\title{
The Noise Performance of a Multiple-Input-Port and Multiple-Output-Port Low-Noise Amplifier Connected to an Array of Coupled Antennas
}

\author{
Frédéric Broydé and Evelyne Clavelier \\ Excem, 12 Chemin des Hauts de Clairefontaine, 78580 Maule, France \\ Correspondence should be addressed to Frédéric Broydé, fredbroyde@eurexcem.com
}

Received 19 May 2011; Revised 17 July 2011; Accepted 18 July 2011

Academic Editor: Hon Tat Hui

Copyright (C) 2011 F. Broydé and E. Clavelier. This is an open access article distributed under the Creative Commons Attribution License, which permits unrestricted use, distribution, and reproduction in any medium, provided the original work is properly cited.

We address the noise characterization of a multiple-input-port and multiple-output-port (MIPMOP) device receiving signals from an array of antennas. A definition of the noise figures and natural noise figures of a MIPMOP device is provided, and the resulting properties are detailed in the impedance and admittance representations. We compute the natural noise figures of a lownoise MIPMOP amplifier comprising a MIMO series-series feedback amplifier (MIMO-SSFA), designed for a wireless receiver front-end.

\section{Introduction}

In this paper, we consider a linear multiple-input-port and multiple-output-port (MIPMOP) device having $n$ input ports and $m$ output ports, connected to an $n$-port source, such as an array of $n$ coupled antennas, and to an $m$ port load. The MIPMOP device could be passive, but our motivation lies in the case where it provides amplification. We want to characterize the noise performance of the MIPMOP device in a small bandwidth $\Delta f$ around a given frequency $f$. The case $n=m=1$ is covered in detail in the literature since more than 50 years $[1,2]$ and will be referred to as the two-port case.

The theory and measurement methods developed for the two-port case can be applied to a MIPMOP device only when all the following conditions are met:

(i) the MIPMOP device is made of $m=n$ uncoupled two-ports;

(ii) the ports of the $n$-port source are uncoupled;

(iii) the ports of the $m$-port source are uncoupled.

The first condition is obvious. However, it is important to note that the theory of the two-port case is irrelevant when the second or the third conditions are not met.
Let us for instance consider the multiport antenna array and the multiport wireless receiver shown in Figure 1, which may be used in a multiple-input and multiple-output (MIMO) or single-input and multiple-output (SIMO) wireless communication system. In Figure $1, \mathbf{Z}_{\text {Sant }}$ is the impedance matrix seen by the receiver, and $Z_{\mathrm{LI}}$ is the input impedance matrix of the receiver. The physical size of the antenna array is often limited by the application, for example in the case of portable transceivers. Antenna coupling may in such a case be so large that the nondiagonal entries cannot be ignored when one computes the directivity patterns and correlations of the channels $[3,4]$. A large coupling between the antennas will also impact the noise performance of the receiver front-end because the noise power delivered by an input port may excite other input ports via antenna coupling.

A conventional wireless receiver front-end uses a MIPMOP device consisting of $n$ uncoupled two-port low-noise amplifiers (LNAs) presenting a diagonal $\mathbf{Z}_{\mathrm{LI}}$. Even though the noise figure of each LNA as a two-port can be defined and measured, it gives no information on the true noise performance of the receiver front-end because it does not take the above-mentioned LNA noise coupled through the antenna array into account. This has been investigated by several authors [5-9]. 


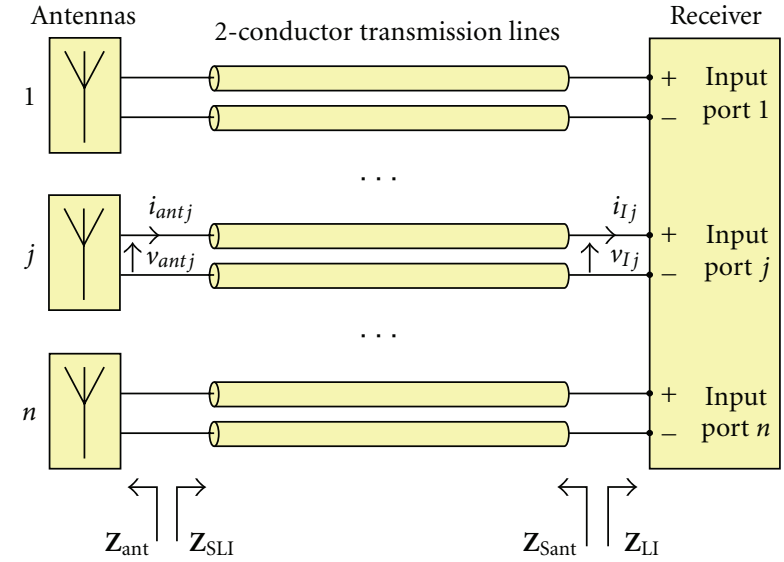

Figure 1: An array of $n$ antennas connected to $n$ uncoupled 2conductor transmission lines connected to a wireless receiver having $n$ input ports.

A type of nonconventional wireless receiver front-end uses a MIPMOP passive matching network to obtain a nondiagonal $\mathbf{Z}_{\mathrm{LI}}$. For instance, a passive lossless MIPMOP device can be used to provide reflectionless matching to its antenna ports (i.e., $\mathbf{Z}_{\mathrm{LI}}=\mathbf{Z}_{\text {Sant }}$ ) and to uncoupled two-port LNAs at its output [10]. More importantly, a passive lossless MIPMOP device can be used to provide hermitian matching to its antenna ports (i.e., $\mathbf{Z}_{\mathrm{LI}}=\mathbf{Z}_{\text {Sant }}^{*}$ where the star denotes the hermitian adjoint) and hermitian matching to uncoupled two-port LNAs at its output [11-14]. This possibility is very interesting since hermitian matching provides a maximum power transfer and decorrelated output voltages in the case of 2-dimensional Rayleigh channels (because the matching network modifies the directional patterns such that they are orthogonal over the azimuth). This type of passive MIPMOP device can also theoretically be used to obtain noise matching $[15,16]$. However, a passive MIPMOP device having a prescribed nondiagonal $Z_{\mathrm{LI}}$ is a complex circuit in which losses must be taken into account to obtain a realistic design $[17,18]$. Such losses degrade the noise performance.

Another type of nonconventional wireless receiver frontend comprises a MIPMOP LNA presenting a nondiagonal $Z_{\mathrm{LI}}$ [19]. This scheme is new [20]. Also, it offers more flexibility than a design using a passive lossless MIPMOP device having its output ports connected to uncoupled twoport LNAs. Thus, better characteristics are expected with an optimized MIPMOP LNA.

In both nonconventional front-end designs, $\mathbf{Z}_{\text {Sant }}$ and $\mathbf{Z}_{\mathrm{LI}}$ are nondiagonal, so that there is no possibility of applying the two-port noise theory to anything. Thus, a noise theory of MIPMOP devices must be used.

In their theory of linear noisy networks, Haus and Adler presented many results which are applicable to MIPMOP devices [21]. However, they did not define an adequate figure of merit, since their purpose was the systematic description of the noise performance of a two-port linear amplifier. More recently, Randa [22] introduced a definition of the noise figures of a MIPMOP device. This definition generalizes the definition used in the two-port case, but it is not directly related to a signal-to-noise ratio.

Randa expressed the noise figures using the S-parameter representation of the device. This formalism is adequate for RF measurements used for the noise characterization of a MIMOP device. However, a noise theory of MIPMOP devices, presented in the impedance and admittance representations, is more appropriate for circuit design. Basic elements of this theory and a validation are available [23]. In this paper, starting from the definitions in Sections 2 and 3 , we further develop this noise theory in Sections 3 to 5 . In Section 6, this theory is applied to the design of a MIPMOP LNA connected to an array of coupled antennas.

\section{Notations}

We use rms values throughout the paper. We use ${ }^{t} \mathbf{X}$ to denote the transpose of a matrix $\mathbf{X}, \overline{\mathbf{X}}$ to denote the complex conjugate of $\mathbf{X}, \mathbf{X}^{*}$ to denote the hermitian adjoint $\mathbf{X}^{*}=$ ${ }^{t} \overline{\mathbf{X}}$, and $\operatorname{tr} \mathbf{X}$ to denote the trace of $\mathbf{X}$.

In the small bandwidth $\Delta f$, we define the column-vector $\mathbf{I}_{\mathrm{IN}}$ of the input noise currents $i_{\mathrm{IN} 1}, \ldots, i_{\mathrm{IN} n}$ flowing into the positive terminals of the input ports of the MIPMOP device and the column vector $\mathbf{V}_{\text {IN }}$ of the input noise voltages $v_{\mathrm{IN} 1}, \ldots, v_{\mathrm{IN} n}$ between the positive and negative terminals of the input ports. We use $\mathbf{I}_{\mathrm{SCIN}}$ and $\mathbf{V}_{\mathrm{OCIN}}$ to denote the column vectors of the short-circuit input noise currents $i_{\mathrm{SCIN}} 1, \ldots, i_{\mathrm{SCIN} n}$ and of the open-circuit input noise voltages $v_{\mathrm{OCIN}} 1, \ldots, v_{\mathrm{OCIN}} n$, respectively. We also define the column vector $\mathbf{I}_{\mathrm{ON}}$ of the output noise currents $i_{\mathrm{ON} 1}, \ldots, i_{\mathrm{ON} m}$ flowing into the positive terminals of the output ports and the column vector $\mathbf{V}_{\mathrm{ON}}$ of the output noise voltages $v_{\mathrm{ON} 1}, \ldots, v_{\mathrm{ON} m}$ between the positive and negative terminals of the output ports. We use $\mathbf{I}_{\mathrm{SCON}}$ and $\mathbf{V}_{\mathrm{OCON}}$ to denote the column vectors of the short-circuit output noise currents $i_{\text {SCON } 1}, \ldots, i_{\text {SCON } m}$ and of the open-circuit output noise voltages $v_{\mathrm{OCON}} 1, \ldots, v_{\mathrm{OCON}}$, respectively. Let us write

$$
\begin{array}{cc}
\mathbf{V}_{N}=\left(\begin{array}{c}
\mathbf{V}_{\mathrm{IN}} \\
\mathbf{V}_{\mathrm{ON}}
\end{array}\right), \quad \mathbf{V}_{\mathrm{OCN}}=\left(\begin{array}{c}
\mathbf{V}_{\mathrm{OCIN}} \\
\mathbf{V}_{\mathrm{OCON}}
\end{array}\right), \\
\mathbf{I}_{N}=\left(\begin{array}{c}
\mathbf{I}_{\mathrm{IN}} \\
\mathbf{I}_{\mathrm{ON}}
\end{array}\right), \quad \mathbf{I}_{\mathrm{SCN}}=\left(\begin{array}{c}
\mathbf{I}_{\mathrm{SCIN}} \\
\mathbf{I}_{\mathrm{SCON}}
\end{array}\right) .
\end{array}
$$

For the case $n=m=2$, we obtain the equivalent circuit $a$ of Figure 2, consisting of $n+m=4$ noise voltage sources and one noiseless $(n+m)$-port. Such an equivalent circuit corresponds to the impedance representation, since

$$
\mathbf{V}_{N}=\mathbf{Z} \mathbf{I}_{N}+\mathbf{V}_{\mathrm{OCN}}
$$

where $\mathbf{Z}$ is the impedance matrix of the MIPMOP device, of size $(n+m) \times(n+m)$. Alternatively, we obtain the equivalent circuit $b$ shown in Figure 2, consisting of $n+m=$ 4 noise current sources and one noiseless $(n+m)$-port. Such an equivalent circuit corresponds to the admittance representation, since

$$
\mathbf{I}_{N}=\mathbf{Y} \mathbf{V}_{N}+\mathbf{I}_{\mathrm{SCN}},
$$




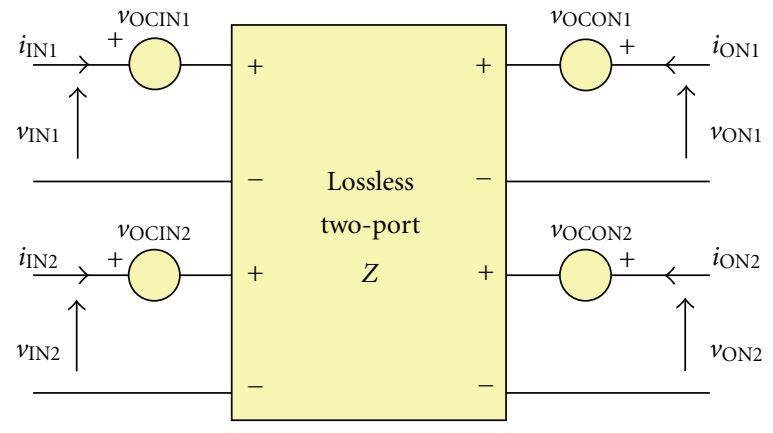

(a)

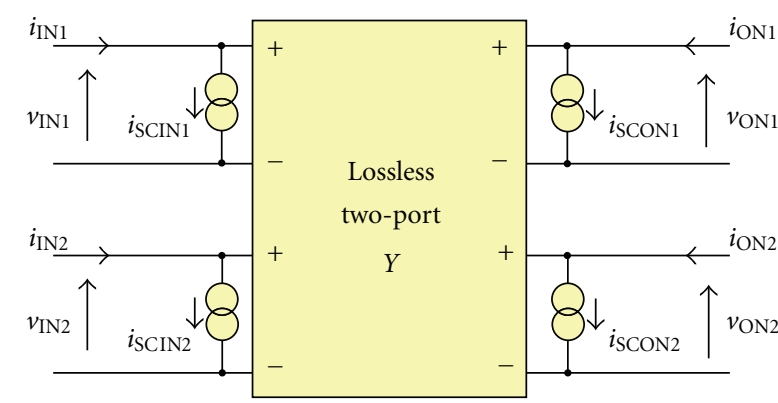

(b)

FIGURE 2: Equivalent circuit for a noisy MIPMOP device.

where $\mathbf{Y}=\mathbf{Z}^{-1}$ is the admittance matrix of the MIPMOP device. We easily obtain

$$
\begin{aligned}
& \mathbf{V}_{\mathrm{OCN}}=-\mathbf{Z} \mathbf{I}_{\mathrm{SCN}}, \\
& \mathbf{I}_{\mathrm{SCN}}=-\mathbf{Y} \mathbf{V}_{\mathrm{OCN}} .
\end{aligned}
$$

The second-order statistics of the noise produced by the MIPMOP device is determined by the covariance matrix of the open-circuit noise voltages $\left\langle\mathbf{V}_{\mathrm{OCN}} \mathbf{V}_{\mathrm{OCN}}^{*}\right\rangle$ or equivalently by the covariance matrix of the short-circuit noise currents $\left\langle\mathbf{I}_{\mathrm{SCN}} \mathbf{I}_{\mathrm{SCN}}^{*}\right\rangle$, where the brackets \langle\rangle indicate an average over an ensemble of noise processes. The covariance matrices are related by

$$
\begin{aligned}
& \left\langle\mathbf{V}_{\mathrm{OCN}} \mathbf{V}_{\mathrm{OCN}}^{*}\right\rangle=\mathbf{Z}\left\langle\mathbf{I}_{\mathrm{SCN}} \mathbf{I}_{\mathrm{SCN}}^{*}\right\rangle \mathbf{Z}^{*}, \\
& \left\langle\mathbf{I}_{\mathrm{SCN}} \mathbf{I}_{\mathrm{SCN}}^{*}\right\rangle=\mathbf{Y}\left\langle\mathbf{V}_{\mathrm{OCN}} \mathbf{V}_{\mathrm{OCN}}^{*}\right\rangle \mathbf{Y}^{*} .
\end{aligned}
$$

These relations are important for noise computations because they can be used to switch between the impedance and admittance representations to easily take into account additional series-connected or shunt-connected circuit elements $[24,25]$.

\section{Noise Figures of MIPMOP Devices}

In the definition of the noise figure of a two-port, the spectral density of the available power of the noise delivered by the source is $k T_{0}$ where $k$ is Boltzmann's constant and $T_{0}=$ $290 \mathrm{~K}$. In the case of devices having multiple input ports, we have to consider that the noises delivered by the different ports of the $n$-port source may be correlated and may have different noise temperatures.

Definition 1. If $\alpha$ is an integer such that $1 \leq \alpha \leq m$, the noise figure $F_{\alpha}$ of the MIPMOP device for the output $\alpha$ is the ratio of the self-power spectral density $\operatorname{Re}\left(\left\langle v_{\mathrm{ON} \alpha} \overline{i_{\mathrm{ON} \alpha}}\right\rangle\right) / \Delta f$ at the output $\alpha$ to the portion of this self-power spectral density that is due to the input noise, evaluated for the case where (a) the available power spectral density of the $n$-port source is $n k T_{0}$ and (b) the available power of the noise delivered by the load is zero.
The noise figures defined above are identical to the one defined by Randa [22] using the scattering representation, except that the condition (a) is not required in his definition. We use this condition because it guaranties that our definition becomes the usual definition in the two-port case, as shown in Section 4.

Let us first assume that the impedance matrix of the source is $\mathbf{Z}_{S}$, that the column vector of the open-circuit noise voltages produced by the source is $\mathbf{E}_{S N}$, and that the impedance matrix of the noiseless load is $Z_{L}$. We have

$$
\mathbf{V}_{N}=\left(\begin{array}{c}
\mathbf{E}_{\mathrm{SN}} \\
\mathbf{0}_{m 1}
\end{array}\right)-\mathbf{Z}_{\mathrm{SL}} \mathbf{I}_{N} \quad \text { with } \mathbf{Z}_{\mathrm{SL}}=\left(\begin{array}{cc}
\mathbf{Z}_{\mathrm{S}} & \mathbf{0}_{n m} \\
\mathbf{0}_{m n} & \mathbf{Z}_{\mathrm{L}}
\end{array}\right),
$$

where $\mathbf{0}_{p q}$ is used to denote the null matrix of size $p \times q$. We use (3) and (7) to write

$$
\mathbf{I}_{N}=\left(\mathbf{Z}+\mathbf{Z}_{\mathrm{SL}}\right)^{-1}\left(\left(\begin{array}{c}
\mathbf{E}_{\mathrm{SN}} \\
\mathbf{0}_{m 1}
\end{array}\right)-\mathbf{V}_{\mathrm{OCN}}\right) .
$$

Consequently, we have

$$
\begin{aligned}
\left\langle\mathbf{I}_{N} \mathbf{I}_{N}^{*}\right\rangle= & \left(\mathbf{Z}+\mathbf{Z}_{\mathrm{SL}}\right)^{-1}\left(\begin{array}{cc}
\left\langle\mathbf{E}_{\mathrm{SN}} \mathbf{E}_{\mathrm{SN}}^{*}\right\rangle & \mathbf{0}_{n m} \\
\mathbf{0}_{m n} & \mathbf{0}_{m m}
\end{array}\right)\left(\mathbf{Z}+\mathbf{Z}_{\mathrm{SL}}\right)^{-1 *} \\
& +\left(\mathbf{Z}+\mathbf{Z}_{\mathrm{SL}}\right)^{-1}\left\langle\mathbf{V}_{\mathrm{OCN}} \mathbf{V}_{\mathrm{OCN}}^{*}\right\rangle\left(\mathbf{Z}+\mathbf{Z}_{\mathrm{SL}}\right)^{-1 *} .
\end{aligned}
$$

By (2), we directly obtain

$$
\left\langle\mathbf{I}_{N} \mathbf{I}_{N}^{*}\right\rangle=\left(\begin{array}{cc}
\left\langle\mathbf{I}_{\mathrm{IN}} \mathbf{I}_{\mathrm{IN}}^{*}\right\rangle & \left\langle\mathbf{I}_{\mathrm{IN}} \mathbf{I}_{\mathrm{ON}}^{*}\right\rangle \\
\left\langle\mathbf{I}_{\mathrm{ON}} \mathbf{I}_{\mathrm{IN}}^{*}\right\rangle & \left\langle\mathbf{I}_{\mathrm{ON}} \mathbf{I}_{\mathrm{ON}}^{*}\right\rangle
\end{array}\right),
$$

so that

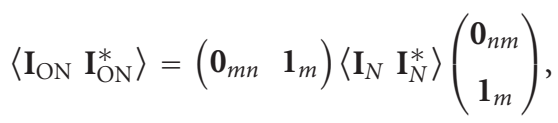

where $\mathbf{1}_{p}$ is the identity matrix of size $p \times p$. Since by (1), (2), and (7) we have $\mathbf{V}_{\mathrm{ON}}=-\mathbf{Z}_{L} \mathbf{I}_{\mathrm{ON}}$, we can write

$$
-\left\langle\mathbf{V}_{\mathrm{ON}} \mathbf{I}_{\mathrm{ON}}^{*}\right\rangle=\left(\begin{array}{ll}
\mathbf{0}_{m n} & \mathbf{Z}_{L}
\end{array}\right)\left\langle\mathbf{I}_{N} \mathbf{I}_{N}^{*}\right\rangle\left(\begin{array}{c}
\mathbf{0}_{n m} \\
\mathbf{1}_{m}
\end{array}\right) .
$$


Using (9) in (12), we obtain the matrix of the self- and cross-complex powers received by the $m$-port load, in the impedance representation

$$
\begin{aligned}
- & \left\langle\mathbf{V}_{\mathrm{ON}} \mathbf{I}_{\mathrm{ON}}^{*}\right\rangle \\
= & \left(\begin{array}{ll}
\mathbf{0}_{m n} & \mathbf{Z}_{L}
\end{array}\right) \\
& \times\left\{\left(\mathbf{Z}+\mathbf{Z}_{\mathrm{SL}}\right)^{-1}\left(\begin{array}{cc}
\left\langle\mathbf{E}_{\mathrm{SN}} \mathbf{E}_{\mathrm{SN}}^{*}\right\rangle & \mathbf{0}_{n m} \\
\mathbf{0}_{m n} & \mathbf{0}_{m m}
\end{array}\right)\left(\mathbf{Z}+\mathbf{Z}_{\mathrm{SL}}\right)^{-1 *}\right. \\
& \left.\quad+\left(\mathbf{Z}+\mathbf{Z}_{\mathrm{SL}}\right)^{-1}\left\langle\mathbf{V}_{\mathrm{OCN}} \mathbf{V}_{\mathrm{OCN}}^{*}\right\rangle\left(\mathbf{Z}+\mathbf{Z}_{\mathrm{SL}}\right)^{-1 *}\right\} \\
& \times\left(\begin{array}{c}
\mathbf{0}_{n m} \\
\mathbf{1}_{m}
\end{array}\right) .
\end{aligned}
$$

Let us use $\mathbf{V}_{\mathrm{SN}}$ and $\mathbf{I}_{\mathrm{SN}}$ to denote the column vectors of noise voltages and noise currents of the source, respectively. In the case of hermitian matching, we have

$$
\begin{gathered}
\mathbf{I}_{\mathrm{SN}}=\left(\mathbf{Z}_{S}+\mathbf{Z}_{S}^{*}\right)^{-1} \mathbf{E}_{\mathrm{SN}}, \\
\mathbf{V}_{\mathrm{SN}}=\mathbf{Z}_{S}^{*}\left(\mathbf{Z}_{S}+\mathbf{Z}_{S}^{*}\right)^{-1} \mathbf{E}_{\mathrm{SN}} .
\end{gathered}
$$

Thus, the matrix of the self- or cross-complex powers which the $n$-port source would deliver to a noiseless $n$-port load providing hermitian matching is

$$
\begin{aligned}
\mathbf{P}_{\mathrm{CSN}} & =\left\langle\mathbf{V}_{\mathrm{SN}} \mathbf{I}_{\mathrm{SN}}^{*}\right\rangle \\
& =\mathbf{Z}_{S}^{*}\left(\mathbf{Z}_{S}+\mathbf{Z}_{S}^{*}\right)^{-1}\left\langle\mathbf{E}_{\mathrm{SN}} \mathbf{E}_{\mathrm{SN}}^{*}\right\rangle\left(\mathbf{Z}_{S}+\mathbf{Z}_{S}^{*}\right)^{-1} .
\end{aligned}
$$

We therefore have

$$
\left\langle\mathbf{E}_{\mathrm{SN}} \mathbf{E}_{\mathrm{SN}}^{*}\right\rangle=\left(\mathbf{Z}_{S}+\mathbf{Z}_{S}^{*}\right) \mathbf{Z}_{S}^{-1 *} \mathbf{P}_{\mathrm{CSN}}\left(\mathbf{Z}_{S}+\mathbf{Z}_{S}^{*}\right) .
$$

The available power of the $n$-port source, denoted by $P_{\mathrm{ASN}}$, is equal to the real power which it delivers to a noiseless $n$-port load providing hermitian matching [12]. The available power of the $n$-port source is

$$
\begin{aligned}
P_{\mathrm{ASN}} & =\operatorname{Re}\left(\left\langle\mathbf{I}_{\mathrm{SN}}^{*} \mathbf{V}_{\mathrm{SN}}\right\rangle\right) \\
& =\operatorname{Re}\left(\left\langle\mathbf{E}_{\mathrm{SN}}^{*}\left(\mathbf{Z}_{S}+\mathbf{Z}_{S}^{*}\right)^{-1} \mathbf{Z}_{S}^{*}\left(\mathbf{Z}_{S}+\mathbf{Z}_{S}^{*}\right)^{-1} \mathbf{E}_{\mathrm{SN}}\right\rangle\right),
\end{aligned}
$$

where we have used (14). Thus,

$$
\begin{aligned}
P_{\mathrm{ASN}} & =\frac{1}{2}\left\langle\mathbf{E}_{\mathrm{SN}}^{*}\left(\mathbf{Z}_{S}+\mathbf{Z}_{S}^{*}\right)^{-1} \mathbf{E}_{\mathrm{SN}}\right\rangle \\
& =\operatorname{Re}\left(\operatorname{tr} \mathbf{P}_{\mathrm{CSN}}\right)=-\operatorname{tr} \mathbf{N}_{\mathrm{Z}},
\end{aligned}
$$

where we have used the characteristic noise matrix of the source in the impedance representation [21, chapter 3], defined as

$$
\mathbf{N}_{Z}=-\frac{1}{2}\left(\mathbf{Z}_{S}+\mathbf{Z}_{S}^{*}\right)^{-1}\left\langle\mathbf{E}_{\mathrm{SN}} \mathbf{E}_{\mathrm{SN}}^{*}\right\rangle .
$$

Consequently, in the impedance representation, the noise figure $F_{\alpha}$ may be written

$$
F_{\alpha}=1+\frac{\operatorname{Re}\left[\left\{\mathbf{Z}_{\mathrm{HL}} \mathbf{Y}_{\mathrm{TL}}\left\langle\mathbf{V}_{\mathrm{OCN}} \mathbf{V}_{\mathrm{OCN}}^{*}\right\rangle \mathbf{Y}_{\mathrm{TL}}^{*}\right\}_{n+\alpha n+\alpha}\right]}{\operatorname{Re}\left[\left\{\mathbf{Z}_{\mathrm{HL}} \mathbf{Y}_{\mathrm{TL}}\left(\begin{array}{cc}
\left\langle\mathrm{E}_{\mathrm{SN}} \mathrm{E}_{\mathrm{SN}}^{*}\right\rangle & \mathbf{0}_{n m} \\
\mathbf{0}_{m n} & \mathbf{0}_{m m}
\end{array}\right) \mathbf{Y}_{\mathrm{TL}}^{*}\right\}_{n+\alpha+\alpha}\right]},
$$

where $\mathbf{Y}_{\mathrm{TL}}=\left(\mathbf{Z}+\mathbf{Z}_{\mathrm{SL}}\right)^{-1}$, and $\{\mathbf{X}\}_{i j}$ is the entry of row $i$ and column $j$ of $\mathbf{X}$, where $\left\langle\mathbf{E}_{\mathrm{SN}} \mathbf{E}_{\mathrm{SN}}^{*}\right\rangle$ is computed for $P_{\mathrm{ASN}}=$ $-\operatorname{tr} \mathbf{N}_{Z}=n k T_{0} \Delta f$ and where

$$
\mathbf{Z}_{\mathrm{HL}}=\left(\begin{array}{cc}
\mathbf{0}_{n n} & \mathbf{0}_{n m} \\
\mathbf{0}_{m n} & \mathbf{Z}_{L}
\end{array}\right) .
$$

Alternatively, we can use $\mathbf{Y}_{S}$ to denote the admittance matrix of the source, $\mathbf{J}_{\mathrm{SN}}$ to denote the column vector of the short-circuit noise currents produced by the source, and $\mathbf{Y}_{L}$ the admittance matrix of the noiseless load. We have

$$
\mathbf{I}_{N}=\left(\begin{array}{c}
\mathbf{J}_{\mathrm{SN}} \\
\mathbf{0}_{m 1}
\end{array}\right)-\mathbf{Y}_{\mathrm{SL}} \mathbf{V}_{N} \quad \text { with } \mathbf{Y}_{\mathrm{SL}}=\left(\begin{array}{cc}
\mathbf{Y}_{S} & \mathbf{0}_{n m} \\
\mathbf{0}_{m n} & \mathbf{Y}_{L}
\end{array}\right) \text {. }
$$

We use (4) and (22) to write

$$
\mathbf{V}_{N}=\left(\mathbf{Y}+\mathbf{Y}_{\mathrm{SL}}\right)^{-1}\left(\left(\begin{array}{c}
\mathbf{J}_{\mathrm{SN}} \\
\mathbf{0}_{m 1}
\end{array}\right)-\mathbf{I}_{\mathrm{SCN}}\right)
$$

Proceeding as above to obtain (13), we get the matrix of the self- and cross-complex powers received by the $m$-port load, in the admittance representation

$$
\begin{aligned}
& -\left\langle\mathbf{V}_{\mathrm{ON}} \mathbf{I}_{\mathrm{ON}}^{*}\right\rangle \\
& =\left(\begin{array}{ll}
\mathbf{0}_{m n} & \mathbf{1}_{m}
\end{array}\right) \\
& \quad \times\left\{\left(\mathbf{Y}+\mathbf{Y}_{\mathrm{SL}}\right)^{-1}\left(\begin{array}{cc}
\left\langle\mathbf{J}_{\mathrm{SN}} \mathbf{J}_{\mathrm{SN}}^{*}\right\rangle & \mathbf{0}_{n m} \\
\mathbf{0}_{m n} & \mathbf{0}_{m m}
\end{array}\right)\left(\mathbf{Y}+\mathbf{Y}_{\mathrm{SL}}\right)^{-1 *}\right. \\
& \left.\quad+\left(\mathbf{Y}+\mathbf{Y}_{\mathrm{SL}}\right)^{-1}\left\langle\mathbf{I}_{\mathrm{OCN}} \mathbf{I}_{\mathrm{OCN}}^{*}\right\rangle\left(\mathbf{Y}+\mathbf{Y}_{\mathrm{SL}}\right)^{-1 *}\right\} \\
& \quad \times\left(\begin{array}{c}
\mathbf{0}_{n m} \\
\mathbf{Y}_{L}^{*}
\end{array}\right) .
\end{aligned}
$$

The matrix of the self- or cross-complex powers which the $n$-port source would deliver to a noiseless $n$-port load providing hermitian matching is given by

$$
\mathbf{P}_{\mathrm{CSN}}=\left(\mathbf{Y}_{S}+\mathbf{Y}_{S}^{*}\right)^{-1}\left\langle\mathbf{J}_{\mathrm{SN}} \mathbf{J}_{\mathrm{SN}}^{*}\right\rangle\left(\mathbf{Y}_{S}+\mathbf{Y}_{S}^{*}\right)^{-1} \mathbf{Y}_{S} .
$$

We therefore have

$$
\left\langle\mathbf{J}_{\text {SN }} \mathbf{J}_{\mathrm{SN}}^{*}\right\rangle=\left(\mathbf{Y}_{S}+\mathbf{Y}_{S}^{*}\right) \mathbf{P}_{\mathrm{CSN}} \mathbf{Y}_{S}^{-1}\left(\mathbf{Y}_{S}+\mathbf{Y}_{S}^{*}\right) .
$$

The available power of the $n$-port source is

$$
\begin{aligned}
P_{\mathrm{ASN}} & =\frac{1}{2}\left\langle\mathbf{J}_{\mathrm{SN}}^{*}\left(\mathbf{Y}_{S}+\mathbf{Y}_{S}^{*}\right)^{-1} \mathbf{J}_{\mathrm{SN}}\right\rangle \\
& =\operatorname{Re}\left(\operatorname{tr} \mathbf{P}_{\mathrm{CSN}}\right)=-\operatorname{tr} \mathbf{N}_{Y},
\end{aligned}
$$


where $\mathbf{N}_{Y}$ is the characteristic noise matrix of the source in the admittance representation, given by

$$
\mathbf{N}_{Y}=-\frac{1}{2}\left(\mathbf{Y}_{S}+\mathbf{Y}_{S}^{*}\right)^{-1}\left\langle\mathbf{J}_{\mathrm{SN}} \mathbf{J}_{S \mathrm{~N}}^{*}\right\rangle .
$$

Thus, in the admittance representation, the noise figure $F_{\alpha}$ is given by

$$
F_{\alpha}=1+\frac{\operatorname{Re}\left[\left\{\mathbf{Z}_{\mathrm{TL}}\left\langle\mathbf{I}_{\mathrm{SCN}} \mathbf{I}_{\mathrm{SCN}}^{*}\right\rangle \mathbf{Z}_{\mathrm{TL}}^{*} \mathbf{Y}_{\mathrm{HL}}^{*}\right\}_{n+\alpha+\alpha}\right]}{\operatorname{Re}\left[\left\{\mathbf{Z}_{\mathrm{TL}}\left(\begin{array}{cc}
\left\langle\mathrm{JSN}_{\mathrm{SN}}^{*}\right\rangle \\
\mathbf{0}_{m n} & \mathbf{0}_{m m}
\end{array}\right) \mathbf{Z}_{\mathrm{TL}}^{*} \mathbf{Y}_{\mathrm{HL}}^{*}\right\}_{n+\alpha+\alpha}\right]},
$$

where $\mathbf{Z}_{\mathrm{TL}}=\left(\mathbf{Y}+\mathbf{Y}_{\mathrm{SL}}\right)^{-1}$ and $\left\langle\mathbf{J}_{\mathrm{SN}} \mathbf{J}_{\mathrm{SN}}^{*}\right\rangle$ is computed for $P_{\mathrm{ASN}}=-\operatorname{tr} \mathbf{N}_{Y}=n k T_{0} \Delta f$ and

$$
\mathbf{Y}_{\mathrm{HL}}=\left(\begin{array}{cc}
\mathbf{0}_{n n} & \mathbf{0}_{n m} \\
\mathbf{0}_{m n} & \mathbf{Y}_{L}
\end{array}\right) \text {. }
$$

\section{Properties of the Noise Figures}

The above-defined noise figures of a MIPMOP device do not depend on the particular representation used to express them, since the definition is based on a ratio of real powers. We also note that $\mathbf{P}_{\mathrm{CSN}}$ and $P_{\mathrm{ASN}}$ do not depend on the representation chosen.

The four following properties indicate that our definition of the noise figures of a MIPMOP device is an adequate generalization of the well-known definition for a two-port.

Property 1. In the case $n=m=1, F_{1}$ is the noise figure $F$ defined for the two-port, which does not depend on $\mathbf{Z}_{L}$ or $\mathbf{Y}_{L}$.

Proof. For $n=m=1$, Definition 1 is the standard definition of $F$ for a two-port [22, Section 2.1].

We note that for $n=1$, using (18), (27) and $P_{\mathrm{ASN}}=$ $k T_{0} \Delta f$, we get the usual single-port noise source formulas

$$
\begin{gathered}
\left\langle\mathbf{E}_{\mathrm{SN}} \mathbf{E}_{\mathrm{SN}}^{*}\right\rangle=\left\langle E_{\mathrm{SN}} \overline{E_{\mathrm{SN}}}\right\rangle=4 k T_{0} \Delta f \operatorname{Re}\left(Z_{S}\right), \\
\left\langle\mathrm{J}_{\mathrm{SN}} \mathrm{J}_{\mathrm{SN}}^{*}\right\rangle=\left\langle J_{\mathrm{SN}} \overline{J_{S N}}\right\rangle=4 k T_{0} \Delta f \operatorname{Re}\left(Y_{S}\right),
\end{gathered}
$$

where the bar indicates the complex conjugate.

Property 2. In the case $n=m$, if the $n$-port source is made of $n$ uncoupled and uncorrelated identical single-port sources of internal impedance $Z_{S}$, if the MIPMOP device is made of $n$ uncoupled identical two-ports having the noise figure $F_{\mathrm{TP}}$ for the source impedance $Z_{S}$, and if the $n$-port load is made of $n$ uncoupled single-port loads of impedance $Z_{L}$, then $F_{\alpha}=F_{\mathrm{TP}}$ for any $\alpha$ such that $1 \leq \alpha \leq m$. Thus, in this case, $F_{\alpha}$ does not depend on $\mathbf{Z}_{L}$ or $\mathbf{Y}_{L}$.

Proof. We use (15) and (19) to write

$$
\begin{aligned}
\mathbf{P}_{\mathrm{CSN}} & =\frac{\overline{Z_{S}}}{4 \operatorname{Re}\left(Z_{S}\right)^{2}}\left\langle\left|E_{\mathrm{SN}}\right|^{2}\right\rangle \mathbf{1}_{n}, \\
\mathbf{N}_{Z} & =-\frac{1}{4 \operatorname{Re}\left(Z_{S}\right)}\left\langle\left|E_{\mathrm{SN}}\right|^{2}\right\rangle \mathbf{1}_{n} .
\end{aligned}
$$

Consequently, for $P_{\mathrm{ASN}}=-\operatorname{tr} \mathrm{N} Z=n k T_{0} \Delta f$, we have

$$
\left\langle\mathbf{E}_{\mathrm{SN}} \mathbf{E}_{\mathrm{SN}}^{*}\right\rangle=4 k T_{0} \Delta f \operatorname{Re}\left(Z_{S}\right) \mathbf{1}_{n} .
$$

Let us use $\mathbf{z}$ to denote the $2 \times 2$ impedance matrix of one of said identical two-ports and $z_{i j}$ to denote an entry of $\mathbf{z}$. We have

$$
\mathbf{Z}+\mathbf{Z}_{\mathrm{SL}}=\left(\begin{array}{cc}
\left(z_{11}+Z_{S}\right) \mathbf{1}_{n} & z_{12} \mathbf{1}_{n} \\
z_{21} \mathbf{1}_{n} & \left(z_{22}+Z_{L}\right) \mathbf{1}_{n}
\end{array}\right)
$$

$$
\left(\mathbf{Z}+\mathbf{Z}_{\mathrm{SL}}\right)^{-1}=\frac{\left(\begin{array}{cc}
\left(z_{22}+Z_{L}\right) \mathbf{1}_{n} & -z_{12} \mathbf{1}_{n} \\
-z_{21} 1_{n} & \left(z_{11}+Z_{S}\right)_{n}
\end{array}\right)}{Z_{L} Z_{S}+z_{11} Z_{L}+z_{22} Z_{S}+z_{11} z_{22}-z_{12} z_{21}} .
$$

We also have

$$
\left\langle\mathbf{V}_{\mathrm{OCN}} \mathbf{V}_{\mathrm{OCN}}^{*}\right\rangle=\left(\begin{array}{cc}
\left\langle\left|v_{\mathrm{OCIN}}\right|^{2}\right\rangle \mathbf{1}_{n} & \left\langle v_{\mathrm{OCIN}} \overline{v_{\mathrm{OCON}}}\right\rangle \mathbf{1}_{n} \\
\left\langle v_{\mathrm{OCON}} \overline{v_{\mathrm{OCIN}}}\right\rangle \mathbf{1}_{n} & \left\langle\left|v_{\mathrm{OCON}}\right|^{2}\right\rangle \mathbf{1}_{n}
\end{array}\right) .
$$

Using (20), (33), (35) and (36), after some derivations, we get

$$
\begin{aligned}
& F_{\alpha}=1
\end{aligned}
$$

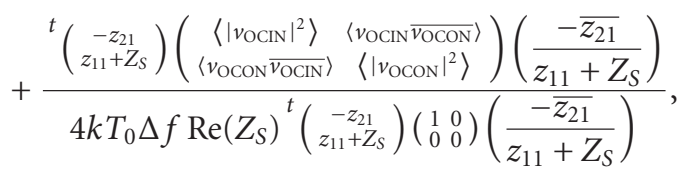

which can be shown to be equal to $F_{\mathrm{TP}}$.

The Property 2 indicates that the noise figures given by (20) and (29) are intensive quantities: each noise figure does not change when the number of identical uncoupled systems is changed.

Property 3. If a MIPMOP device B is made of a cascade consisting of a lossless passive input network having $n$ input ports and $n$ output ports, of a MIPMOP device A having $n$ input ports and $m$ output ports and of a lossless passive output network, then the sum of the noise figures of the MIPMOP device $\mathrm{B}$ is equal to the sum of the noise figures of the MIPMOP device A.

Proof. This is the direct consequence of the following facts:

(i) the lossless passive output network does not change the total real power delivered to the $m$-port load;

(ii) the lossless passive input network does not change the available power of the $n$-port source because it is possible to prove (see the appendix) that hermitian matching occurs at its input ports if and only if hermitian matching occurs at its output ports. 
Property 4. In the configuration considered in the Property 3, if we also require that the lossless passive output network is made of $m$ uncoupled lossless passive two-ports, each having an input port connected to the output port $\alpha$ of the MIPMOP device A and an output port corresponding to the output port $\alpha$ of the MIPMOP device $\mathrm{B}$, then, for any $\alpha$ such that $1 \leq \alpha \leq m$, the noise figure of the MIPMOP device B for the output $\alpha$ is equal to the noise figure of the MIPMOP device A for the output $\alpha$.

Proof. Each lossless passive two-port does not change the real power delivered to each port of the $m$-port load.

The Property 4 is a generalization to MIPMOP devices of a property of noisy two-ports which is a direct consequence of the Friis formula for cascaded noisy two-ports $[1,2]$. We note that the configurations considered in the properties 3 and 4 can be used for computing the noise figures of a MIPMOP amplifier consisting of an active MIPMOP circuit inserted between two MIPMOP matching circuits having negligible losses.

Notwithstanding the fact that $F$ is a vector, we have to keep in mind that there are three important differences between the usual definition of the noise figure for a two-port and the definition introduced in Section 3 for a MIPMOP device:

(i) for $n \geq 2$, each $F_{\alpha}$ depends on the correlations between the ports of the $n$-port source;

(ii) for $m \geq 2$, each $F_{\alpha}$ depends on the $m$-port load, that is to say, on $\mathbf{Z}_{L}$ or on $\mathbf{Y}_{L}$ in the case of a linear $m$-port load [23, Section 3];

(iii) since there is no obvious generalization of the concept of signal-to-noise ratio to MIPMOP devices, the noise figures of a MIPMOP device cannot be viewed as a direct measure of the degradation of a signal-to-noise ratio.

\section{Natural Noise Figures of a MIPMOP Device}

We are going to introduce a definition of the natural noise figures of the MIPMOP device, based on two properties.

Property 5. If the $n$-port source is a noisy passive linear network at the temperature $T$, then the available power spectral density of the $n$-port source is $n k T$. Additionally, we have

$$
\begin{aligned}
& \left\langle\mathbf{E}_{\mathrm{SN}} \mathbf{E}_{\mathrm{SN}}^{*}\right\rangle=2 k T \Delta f\left(\mathbf{Z}_{S}+\mathbf{Z}_{S}^{*}\right), \\
& \left\langle\mathbf{J}_{\mathrm{SN}} \mathbf{J}_{\mathrm{SN}}^{*}\right\rangle=2 k T \Delta f\left(\mathbf{Y}_{S}+\mathbf{Y}_{S}^{*}\right) .
\end{aligned}
$$

Proof. The covariance matrix $\left\langle\mathbf{E}_{\mathrm{SN}} \mathbf{E}_{\mathrm{SN}}^{*}\right\rangle$ is hermitian and positive definite, and $\mathbf{Z}_{S}+\mathbf{Z}_{S}^{*}$ is hermitian. Consequently [26, Section 7.6.5], there exists a nonsingular matrix $\mathbf{C}$ such that $\mathbf{C}^{*}\left(\mathbf{Z}_{S}+\mathbf{Z}_{S}^{*}\right) \mathbf{C}$ is real and diagonal and $\mathbf{C}^{*}\left\langle\mathbf{E}_{\mathrm{SN}} \mathbf{E}_{\mathrm{SN}}^{*}\right\rangle \mathbf{C}=\mathbf{1}_{n}$.

As pointed out in [21, page 29], this transformation may be obtained with a lossless imbedding. We may introduce the impedance matrix $\mathbf{Z}_{S}^{\prime}$ of the resulting network, the column vector of its open-circuit noise voltages $\mathbf{E}_{\mathrm{SN}}^{\prime}$ and write

$$
\begin{aligned}
\mathbf{Z}_{S}^{\prime}+\mathbf{Z}_{S}^{\prime *} & =\mathbf{C}^{*}\left(\mathbf{Z}_{S}+\mathbf{Z}_{S}^{*}\right) \mathbf{C}=2 \operatorname{diag}_{n}\left(R_{1}, \ldots, R_{n}\right), \\
& \left\langle\mathbf{E}_{\mathrm{SN}}^{\prime} \mathbf{Z}_{\mathrm{SN}}^{\prime *}\right\rangle=\mathbf{C}^{*}\left\langle\mathbf{E}_{\mathrm{SN}} \mathbf{E}_{\mathrm{SN}}^{*}\right\rangle \mathbf{C}=\mathbf{1}_{n},
\end{aligned}
$$

where $\operatorname{diag}_{n}\left(x_{1}, \ldots, x_{n}\right)$ denotes the diagonal matrix of size $n \times n$ having the entries $x_{1}, \ldots, x_{n}$, and where $R_{1}, \ldots, R_{n}$ are real. We then introduce the impedance matrix $\mathbf{Z}_{\text {rem }}$ such that

$$
\mathbf{Z}_{S}^{\prime}=\operatorname{diag}_{n}\left(R_{1}, \ldots, R_{n}\right)+\mathbf{Z}_{\text {rem }} .
$$

If we use (42) in (40), we find that

$$
\mathbf{Z}_{\mathrm{rem}}=-\mathbf{Z}_{\mathrm{rem}}^{*}
$$

Thus, $\mathbf{Z}_{\text {rem }}$ is the impedance matrix of a lossless network. We now connect a lossless network of impedance matrix $-\mathbf{Z}_{\text {rem }}$ in series with the network of impedance matrix $\mathbf{Z}_{S}^{\prime}$ and obtain a network of impedance matrix

$$
\mathbf{Z}_{S}^{\prime \prime}=\mathbf{Z}_{S}^{\prime}-\mathbf{Z}_{\text {rem }}=\operatorname{diag}_{n}\left(R_{1}, \ldots, R_{n}\right),
$$

this network having the column vector of the open-circuit noise voltages $\mathbf{E}_{\mathrm{SN}}^{\prime}$. We consequently have $n$ uncoupled resistances at the temperature $T$, which proves that $R_{1}, \ldots, R_{n}$ are positive and

$$
\left\langle\mathbf{E}_{\mathrm{SN}}^{\prime} \mathbf{E}_{\mathrm{SN}}^{\prime *}\right\rangle=n k T \Delta f \operatorname{diag}_{n}\left(R_{1}, \ldots, R_{n}\right) .
$$

Using (41), we obtain

$$
\begin{aligned}
\left\langle\mathbf{E}_{\mathrm{SN}} \mathbf{E}_{\mathrm{SN}}^{*}\right\rangle & =\mathbf{C}^{*-1}\left\langle\mathbf{E}_{\mathrm{SN}}^{\prime} \mathbf{E}_{\mathrm{SN}}^{\prime *}\right\rangle \mathbf{C}^{-1} \\
& =4 n k T \Delta f \mathbf{C}^{*-1} \operatorname{diag}_{n}\left(R_{1}, \ldots, R_{n}\right) \mathbf{C}^{-1}
\end{aligned}
$$

Using (40) we then find that

$$
\begin{aligned}
\left\langle\mathbf{E}_{\mathrm{SN}} \mathbf{E}_{\mathrm{SN}}^{*}\right\rangle & =2 n k T \Delta f\left(\mathbf{Z}_{S}+\mathbf{Z}_{S}^{*}\right), \\
\left\langle\mathbf{J}_{\mathrm{SN}} \mathbf{J}_{\mathrm{SN}}^{*}\right\rangle & =\mathbf{Z}_{S}^{-1}\left\langle\mathbf{E}_{\mathrm{SN}} \mathbf{E}_{\mathrm{SN}}^{*}\right\rangle \mathbf{Z}_{S}^{*-1} \\
& =2 n k T \Delta f\left(\mathbf{Y}_{S}+\mathbf{Y}_{S}^{*}\right) .
\end{aligned}
$$

Property 6. If the $n$-port source produces the noise of a noisy passive network at the temperature $T_{0}$, the constraint (a) of the Definition 1 is automatically satisfied.

Proof. If we use (15), (18), and (38), we find that

$$
\begin{gathered}
\mathbf{P}_{\mathrm{CSN}}=2 k T_{0} \Delta f \mathbf{Z}_{S}^{*}\left(\mathbf{Z}_{S}+\mathbf{Z}_{S}^{*}\right)^{-1}, \\
P_{\mathrm{ASN}}=-\operatorname{tr} \mathbf{N}_{Z}=n k T_{0} \Delta f,
\end{gathered}
$$

which proves the property. Alternatively, we could have used (25), (27), and (39), to obtain

$$
\begin{gathered}
\mathbf{P}_{\mathrm{CSN}}=2 k T_{0} \Delta f\left(\mathbf{Y}_{S}+\mathbf{Y}_{S}^{*}\right)^{-1} \mathbf{Y}_{S}, \\
P_{\mathrm{ASN}}=-\operatorname{tr} \mathbf{N}_{Y}=n k T_{0} \Delta f,
\end{gathered}
$$

and reached the same conclusion. 
Definition 2. If the $n$-port source produces the noise of a noisy passive network at the temperature $T_{0}$, the noise figures of Definition 1 will be referred to as the natural noise figures.

In the laboratory, it is possible to use a nearly ideal noisy passive network at the temperature $T_{0}$, and to measure natural noise figures in this manner. However, this parameter does not necessarily correspond to the application situation. For instance the noise collected by multiple antennas will in general not comply with (48) and (49).

The following property relates to a special case where the natural noise figures are easier to determine using standard measuring instruments. Let us use

(i) $F_{\alpha \beta}^{\prime}$ to denote the noise figure between an input $\beta$ and an output $\alpha$, defined as the usual noise figure of a two-port, evaluated in the case where all unused inputs and outputs are connected to uncoupled and uncorrelated single-port terminations for which the available power spectral density is $k T_{0}$;

(ii) $F_{\alpha \alpha^{\prime}}^{\prime \prime}$ to denote the noise figure between an output $\alpha^{\prime}$, used as an input, and an output $\alpha$, defined as the usual noise figure of a two-port, evaluated in the case where all unused inputs and outputs are connected to uncoupled and uncorrelated single-port terminations for which the available power spectral density is $k T_{0}$.

Property 7. In the case where the $n$-port source is made of uncoupled and uncorrelated single-port sources, and where the $m$-port load is made of uncoupled and uncorrelated single-port loads, the natural noise figures are given by

$$
F_{\alpha}=\frac{1-\sum_{\alpha^{\prime}=1, \alpha^{\prime} \neq \alpha}^{m} 1 / F_{\alpha \alpha^{\prime}}^{\prime \prime}}{\sum_{\beta=1}^{n} 1 / F_{\alpha \beta}^{\prime}}
$$

Proof. All single-port sources and loads being assumed uncoupled and uncorrelated to each other, let us use

(i) $P_{1 \alpha}$ to denote the self-power spectral density at the output $\alpha$, evaluated for the case where (c) the inputs are connected to single-port sources, (d) the available power spectral density of each single-port source is $k T_{0}$, (e) the outputs are connected to single-port loads, and (f) the available power spectral density of each single-port load is zero;

(ii) $P_{2 \alpha \beta}$ to denote the self-power spectral density at the output $\alpha$, evaluated for the case where (g) the MIPMOP device is noiseless, (c) the inputs are connected to single-port sources, (h) the available power spectral density of each single-port source is zero except the single-port source connected to the input $\beta$ for which the available power spectral density is $k T_{0}$, (e) the outputs are connected to single-port loads, and (f) the available power spectral density of each single-port load is zero.

In the case defined above, we have

$$
F_{\alpha}=\frac{P_{1 \alpha}}{\sum_{\beta=1}^{n} P_{2 \alpha \beta}},
$$

so that

$$
F_{\alpha}=\frac{1}{\sum_{\beta=1}^{n} 1 / F_{\alpha \beta}} \quad \text { where } F_{\alpha \beta}=\frac{P_{1 \alpha}}{P_{2 \alpha \beta}} .
$$

$F_{\alpha \beta}$ is not the usual noise figure of a two-port, evaluated in the case where all unused inputs and outputs are connected to single-port terminations, because, in this case, the unused single-port loads also contribute to the self-power spectral density at the output $\alpha$. Let us use $P_{3 \alpha \alpha^{\prime}}$ to denote the self-power spectral density at the output $\alpha$, evaluated for the case where (g) the MIPMOP device is noiseless, (c) the inputs are connected to single-port sources, $(j)$ the available power spectral density of each single-port source is zero, (e) the outputs are connected to single-port loads, and (k) the available power spectral density of each single-port load is zero except the single-port load connected to the output $\alpha^{\prime}$ for which the available power spectral density is $k T_{0}$. We have

$$
\begin{aligned}
F_{\alpha \beta}^{\prime} & =\frac{P_{1 \alpha}+\sum_{\alpha^{\prime}=1, \alpha^{\prime} \neq \alpha}^{m} P_{3 \alpha \alpha^{\prime}}}{P_{2 \alpha \beta}}, \\
F_{\alpha \alpha^{\prime \prime}}^{\prime \prime} & =\frac{P_{1 \alpha}+\sum_{\alpha^{\prime}=1, \alpha^{\prime} \neq \alpha}^{m} P_{3 \alpha \alpha^{\prime}}}{P_{3 \alpha \alpha^{\prime \prime}}} .
\end{aligned}
$$

So that we obtain

$$
F_{\alpha \beta}=F_{\alpha \beta}^{\prime}\left(1-\sum_{\substack{\alpha^{\prime}=1 \\ \alpha^{\prime} \neq \alpha}}^{m} \frac{1}{F_{\alpha \alpha^{\prime}}^{\prime \prime}}\right) .
$$

Using (54) in (52) leads us to (50).

Note that in the case where the contribution of the singleport loads to the self-power spectral density at the output $\alpha$ is negligible, that is,

$$
\sum_{\substack{\alpha^{\prime}=1 \\ \alpha^{\prime} \neq \alpha}}^{m} \frac{1}{F_{\alpha \alpha^{\prime}}^{\prime \prime}} \ll 1,
$$

we may assume that

$$
F_{\alpha} \approx \frac{1}{\sum_{\beta=1}^{n} 1 / F_{\alpha \beta}^{\prime}} .
$$

In [23], (56) was incorrectly presented as an exact formula. However, (56) was a good approximation of the exact formula (50) for the MIPMOP amplifier considered in [23].

\section{A MIPMOP LNA Connected to an Antenna Array}

We will now apply our results to the $1880 \mathrm{MHz}$ MIPMOP LNA shown in Figure 3, having $n=4$ signal input terminals and $m=4$ signal output terminals. It is intended to be used as a wireless receiver front-end. The core of this MIPMOP LNA is a new circuit called MIMO series-series feedback amplifier (MIMO-SSFA) described below [23, 27, 28]. 


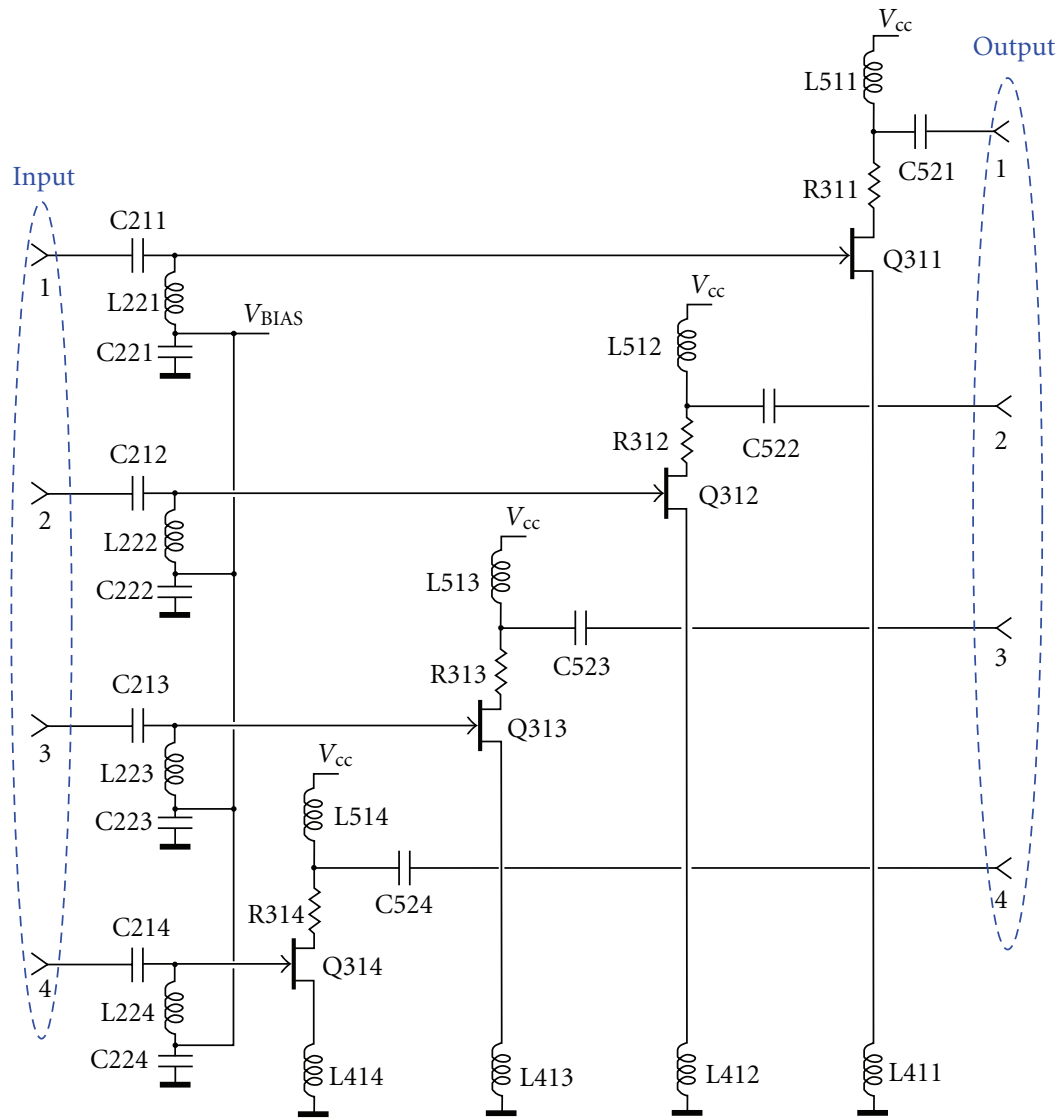

FIGURE 3: A low-noise MIPMOP amplifier comprising a MIMO-SSFA, 4 uncoupled input matching networks, and 4 uncoupled output matching networks.

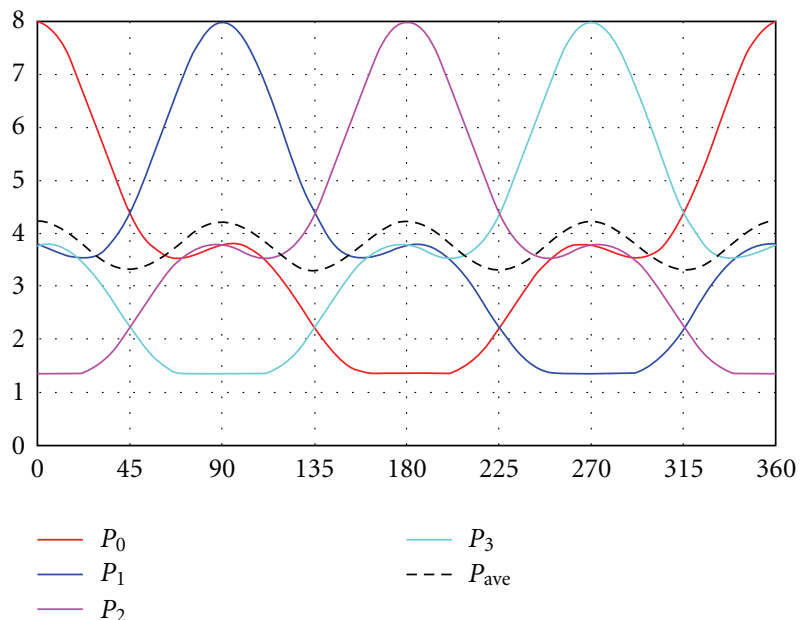

Figure 4: The powers $P 0, P 1, P 2$, and $P 3$ delivered by each antenna (in $\mu \mathrm{W}$ ) and the average power as a function of the azimuth (in degree) for a conventional front-end design receiving an incident plane wave of $1 \mathrm{~V} / \mathrm{m}$ (peak).

Referring to the impedance matrices defined in Figure 1, the MIPMOP LNA is designed to provide hermitian matching between $\mathbf{Z}_{\text {Sant }}$ and $\mathbf{Z}_{\mathrm{LI}}$.

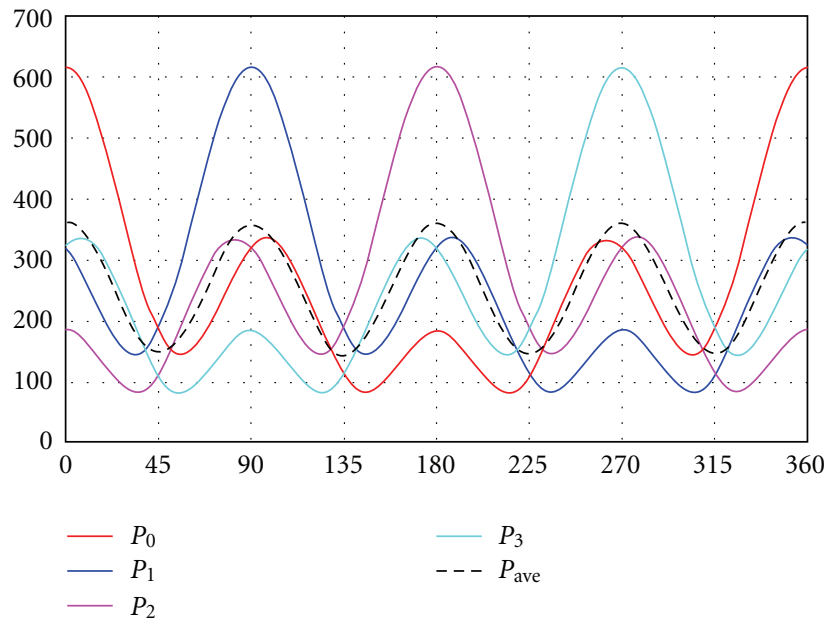

Figure 5: The powers $P 0, P 1, P 2$, and $P 3$ delivered by the outputs of the MIPMOP amplifier shown in Figure 3 (in $\mu \mathrm{W}$ ) and the average power as a function of the azimuth (in degree) when the antenna array receives an incident plane wave of $1 \mathrm{~V} / \mathrm{m}$ (peak).

The antenna array is a circular array of four parallel half-wave dipole antennas (side-by-side configuration) for $1880 \mathrm{MHz}$. The radius of the array is $0.3 \lambda=47.8 \mathrm{~mm}$, 
so that it presents a $0.424 \lambda=67.7 \mathrm{~mm}$ spacing between the nearest array elements. This antenna array has been presented in [19]. The impedance matrix of the antenna array is computed as

$$
\mathbf{Z}_{\mathrm{ant}}=\left(\begin{array}{cccc}
73.1 & 1.1-36.4 j & -23.3-15.9 j & 1.1-36.4 j \\
1.1-36.4 j & 73.1 & 1.1-36.4 j & -23.3-15.9 j \\
-23.3-15.9 j & 1.1-36.4 j & 73.1 & 1.1-36.4 j \\
1.1-36.4 j & -23.3-15.9 j & 1.1-36.4 j & 73.1
\end{array}\right) \Omega \text {. }
$$

For a zenithal angle $\theta=\pi / 2$, and if we assume that the array sees four uncoupled scalar loads of $73.0 \Omega$, the power delivered by each antenna and the average power are shown in Figure 4 as a function of the azimuth for an incident field of $1 \mathrm{~V} / \mathrm{m}$ peak. The average power oscillates between $3.3 \mu \mathrm{W}$ and $4.2 \mu \mathrm{W}$ as a function of the angle of arrival whereas, in the same field, a single dipole antenna in free space would deliver $4.4 \mu \mathrm{W}$ to a $73.0 \Omega$ load.

We assume that each antenna is connected to the MIPMOP amplifier via a two-conductor interconnection (for instance a coaxial cable) behaving as a two-conductor transmission line. The antenna number $j$ is connected to the near-end of the interconnection number $j$ of length $d_{j}$, characteristic impedance $\mathrm{z}_{C j}$, phase velocity $c_{j}$, and attenuation constant $\alpha_{j}$. The far-end of the interconnection number $j$ is connected to the input port number $j$ of the MIPMOP amplifier. Let us define the characteristic impedance matrix $\mathbf{Z}_{C}$ of the set of interconnections as

$$
\mathrm{Z}_{C}=\operatorname{diag}_{n}\left(\mathrm{z}_{C 1}, \ldots, \mathrm{z}_{C n}\right)
$$

Let us also define the transmission matrix $\mathbf{T}$ of the set of interconnections as

$$
\mathbf{T}=\operatorname{diag}_{n}\left(e^{\left.-\left[\alpha_{1}+j\left(2 \pi f_{0} / c_{1}\right)\right)\right] d_{1}}, \ldots, e^{-\left[\alpha_{n}+j\left(2 \pi f_{0} / c_{n}\right)\right] d_{n}}\right)
$$

After some derivation [19], we get

$$
\begin{aligned}
\mathbf{Z}_{\text {Sant }}= & \left\{\frac{\mathbf{T}+\mathbf{T}^{-1}}{2} \mathbf{Z}_{\text {ant }}-\frac{\mathbf{T}-\mathbf{T}^{-1}}{2} \mathbf{Z}_{C}\right\} \\
& \times\left\{\frac{\mathbf{T}+\mathbf{T}^{-1}}{2} \mathbf{Z}_{C}-\frac{\mathbf{T}-\mathbf{T}^{-1}}{2} \mathbf{Z}_{\text {ant }}\right\}^{-1} \mathbf{Z}_{C} .
\end{aligned}
$$

If we assume that four identical $54 \mathrm{~mm}$-long interconnections are used, having, at $f_{0}=1880 \mathrm{MHz}$, a propagation velocity of $0.6 c_{0}$, a characteristic impedance of $80 \Omega$, and an attenuation of $1.667 \mathrm{~dB} / \mathrm{m}$, the resulting impedance matrix seen by the MIPMOP LNA at $f_{0}$ is equal to

$$
\mathbf{Z}_{\text {Sant }}=\left(\begin{array}{cccc}
84.4+10.1 j & -18.7-32.5 j & -17.9+13.5 j & -18.7-32.5 j \\
-18.7-32.5 j & 84.4+10.1 j & -18.7-32.5 j & -17.9+13.5 j \\
-17.9+13.5 j & -18.7-32.5 j & 84.4+10.1 j & -18.7-32.5 j \\
-18.7-32.5 j & -17.9+13.5 j & -18.7-32.5 j & 84.4+10.1 j
\end{array}\right) \Omega
$$

We note that if we compare (57) and (61), the relative difference between diagonal entries is small, but the nondiagonal entries are thoroughly modified.

The MIMO-SSFA consists of $n$ active subcircuits (ASC) and a feedback network. Each ASC comprises a packaged low-noise pseudomorphic HEMT (Q311 to Q314) and a drain resistor ( $\mathrm{R} 311$ to $\mathrm{R} 314$ ) providing resistive loading to improve stability. The feedback network consists of the 4 inductors L411 to L414, coupled by mutual induction. This coupling is the only phenomenon leading to a nondiagonal input impedance matrix $\mathbf{Z}_{\mathrm{LI}}$. The MIPMOP LNA also comprises an input matching network (C211 to C214 and L221 to L224) and an output matching network (C521 to C524 and L511 to L514).

The MIMOP LNA shown in Figure 3 has been designed in such a way that when each output port is connected to a grounded $50 \Omega$ resistor, the amplifier provides an impedance $\mathbf{Z}_{\mathrm{LI}}$ close to $\mathbf{Z}_{\text {Sant }}^{*}\left[29\right.$, Section 5]. At $f_{0}=1880 \mathrm{MHz}$, the resulting voltage gain matrix $\mathbf{G}_{V}$ of the MIPMOP amplifier and $\mathbf{Z}_{\mathrm{LI}}$ are

$$
\mathbf{G}_{V}=\left(\begin{array}{cccc}
-2.80-3.32 j & 0.06+0.86 j & 0.24+0.37 j & 0.06+0.86 j \\
0.06+0.86 j & -2.80-3.32 j & 0.06+0.86 j & 0.24+0.37 j \\
0.24+0.37 j & 0.06+0.86 j & -2.80-3.32 j & 0.06+0.86 j \\
0.06+0.86 j & 0.24+0.37 j & 0.06+0.86 j & -2.80-3.32 j
\end{array}\right),
$$




$$
\mathbf{Z}_{\mathrm{LI}}=\left(\begin{array}{cccc}
84.5-10.2 j & -18.7+32.5 j & -17.9-13.5 j & -18.7+32.5 j \\
-18.7+32.5 j & 84.5-10.2 j & -18.7+32.5 j & -17.9-13.5 j \\
-17.9-13.5 j & -18.7+32.5 j & 84.5-10.2 j & -18.7+32.5 j \\
-18.7+32.5 j & -17.9-13.5 j & -18.7+32.5 j & 84.5-10.2 j
\end{array}\right) \Omega .
$$

The comparison of (61) and (63) shows that hermitian matching is almost exactly reached between the receiver's end of the interconnections and the inputs of the MIPMOP LNA. We computed the power delivered to each $50 \Omega$ resistor and the average power shown in Figure 5, in the configuration used for Figure 4 [19, Section 8.2].

Assuming that the input and output matching networks are lossless, we have computed the noise figures for three configurations of the MIPMOP amplifier, in a worksheet of a generic calculation program. A technique used several times in this worksheet is the following: (6) being possibly used to obtain the appropriate representation, the covariance matrix of a series or parallel connection of two uncorrelated circuits is obtained as a sum of covariance matrices [24, 25].

In this manner we successively determined:

(1) the covariance matrix of the open-circuit voltages $\left\langle\mathbf{V}_{A} \mathbf{V}_{A}^{*}\right\rangle$ of an ASC comprising a transistor and a series drain resistor, this matrix being denoted by

$$
\left\langle\mathbf{V}_{A} \mathbf{V}_{A}^{*}\right\rangle=\left(\begin{array}{ll}
\left\langle V_{A 1} \overline{V_{A 1}}\right\rangle & \left\langle V_{A 1} \overline{V_{A 2}}\right\rangle \\
\left\langle V_{A 2} \overline{V_{A 1}}\right\rangle & \left\langle V_{A 2} \overline{V_{A 2}}\right\rangle
\end{array}\right) ;
$$

(2) the covariance matrix $\left\langle\mathbf{V}_{B} \mathbf{V}_{B}^{*}\right\rangle$ of the open-circuit voltages of the MIMO-SSFA, using

$$
\begin{aligned}
\left\langle\mathbf{V}_{B} \mathbf{V}_{B}^{*}\right\rangle= & \left(\begin{array}{ll}
\left\langle\mathbf{E}_{\mathrm{FB}} \mathbf{E}_{\mathrm{FB}}^{*}\right\rangle & \left\langle\mathbf{E}_{\mathrm{FB}} \mathbf{E}_{\mathrm{FB}}^{*}\right\rangle \\
\left\langle\mathbf{E}_{\mathrm{FB}} \mathbf{E}_{\mathrm{FB}}^{*}\right\rangle & \left\langle\mathbf{E}_{\mathrm{FB}} \mathbf{E}_{\mathrm{FB}}^{*}\right\rangle
\end{array}\right) \\
& +\left(\begin{array}{cc}
\left\langle V_{A 1} \overline{V_{A 1}}\right\rangle \mathbf{1}_{n} & \left\langle V_{A 1} \overline{V_{A 2}}\right\rangle \mathbf{1}_{n} \\
\left\langle V_{A 2} \overline{V_{A 1}}\right\rangle_{n} & \left\langle V_{A 2} \overline{V_{A 2}}\right\rangle_{n}
\end{array}\right),
\end{aligned}
$$

and the assumption that the feedback network of the MIMO-SSFA behaves as a noisy passive network at the temperature $T_{0}$, so that according to (38) the covariance matrix of the open circuit voltages at the terminals of the feedback network is

$$
\left\langle\mathbf{E}_{\mathrm{FB}} \mathbf{E}_{\mathrm{FB}}^{*}\right\rangle=2 k T_{0} \Delta f\left(\mathbf{Z}_{\mathrm{FB}}+\mathbf{Z}_{\mathrm{FB}}^{*}\right),
$$

where $\mathrm{Z}_{\mathrm{FB}}$ is the impedance matrix of the feedback network, the real part of which is determined by losses in the inductors L411 to L414;

(3) the noise figures of the MIMO-SSFA, using (20) applied to $\left\langle\mathbf{V}_{A} \mathbf{V}_{A}^{*}\right\rangle$ given by (65);

(4) the matrices $\left\langle\mathbf{V}_{\mathrm{OCN}} \mathbf{V}_{\mathrm{OCN}}^{*}\right\rangle$ and $\left\langle\mathbf{I}_{\mathrm{SCN}} \mathbf{I}_{\mathrm{SCN}}^{*}\right\rangle$ of the MIPMOP LNA, once parallel and series circuit elements of the input and output matching networks are included;
(5) the noise figures of the MIPMOP LNA, using (20) applied to $\left\langle\mathbf{V}_{\text {OCN }} \mathbf{V}_{\text {OCN }}^{*}\right\rangle$ or (29) applied to $\left\langle\mathbf{I}_{\mathrm{SCN}} \mathbf{I}_{\mathrm{SCN}}^{*}\right\rangle$.

Since we assume that the input and output matching networks are lossless, we expect that the steps (3) and (5) provide the same result, according to the Property 4 . We indeed always obtain the same noise figures, in the three configurations defined below.

In the first configuration, we have canceled the mutual induction between the inductors L411 to L414, the resulting MIPMOP amplifier being driven by a source having a diagonal impedance matrix equal to $(84.436+j 10.124) \mathbf{1}_{n} \Omega$. Consequently, the four channels are uncoupled. All computed natural noise figures are equal to about $1.134 \mathrm{~dB}$, irrespective of the choice of diagonal load impedance matrix. We checked that in line with the Property 2, this result is the same as the one obtained for one channel treated as a twoport.

In the second configuration, we have kept zero mutual inductances between the inductors L411 to L414, but the input of the resulting MIPMOP amplifier sees the impedance matrix $\mathbf{Z}_{\text {Sant }}$ given by (61). All computed natural noise figures are equal to about $1.218 \mathrm{~dB}$, for a load impedance matrix equal to $501_{m} \Omega$. Consequently, the couplings between the antennas increase the noise figures, as qualitatively explained in the introduction. We also observed that, as expected, these noise figures depend on the choice of the diagonal load impedance matrix.

In the third configuration, the mutual inductances between the inductors L411 to L414 had their nominal values, the MIPMOP amplifier being driven by a source having an impedance matrix equal to $\mathbf{Z}_{\text {Sant }}$ given by (61). This configuration is the MIPMOP LNA designed in [29] and used in [19], as said above. In this configuration, all natural noise figures are equal to about $1.896 \mathrm{~dB}$, for a load impedance matrix equal to $50 \mathbf{1}_{m} \Omega$. We again observed that, as expected, these noise figures depend on the choice of the diagonal load impedance matrix.

It must again be emphasized that, for the second and third configurations, there is no direct relationship between the computed natural noise figures and a measure of the degradation of a signal-to-noise ratio, as pointed out in the introduction and at the end of Section 4.

\section{Conclusion}

In this paper, we have defined the noise figures of a MIPMOP device in a manner that extends the classical definition applicable to a two-port. This definition has an additional 
condition compared to the definition used by Randa [22]. When the $n$-port source connected to the input ports of the MIPMOP device is a passive multiport source at $T_{0}$, our noise figures are referred to as natural noise figures, and the source impedance matrix determines the self- and crosscorrelations of the noises applied to the input ports. We have also developed a theory of the noise figures of MIPMOP devices in the impedance and admittance representations.

We have presented an example which shows how this theory can be applied to the design of a MIPMOP LNA connected to an array of coupled antennas and providing hermitian matching. It is worth noting that if mutual induction was not present, Figure 3 would represent four independent single-input and single-output LNA with inductive source degeneration. This scheme is popular because it can simultaneously provide a high gain and a low noise figure [30-33]. Since introducing mutual induction between coils does not involve any additional circuit element, the 4-input and 4-output MIPMOP LNA shown in Figure 3 has the same number of circuit elements as four independent 2-port LNAs with inductive source degeneration.

In the example treated in Section 6, we observe that $\mathbf{Z}_{C}$ given by (58) is quite different from $\mathbf{Z}_{\mathrm{LI}}$ given by (63) and $\mathbf{Z}_{\text {ant }}$ given by (57), so that multiple reflections will take place. This phenomenon is inherent to hermitian matching. It does not cause problems when the interconnections between the antennas and the MIPMOP LNA are sufficiently short.

\section{Appendix}

\section{A Theorem on Linear MIPMOP Devices}

A linear MIPMOP device having $n$ input ports and $m$ output ports is characterized by the admittance matrices $\mathbf{Y}_{\mathrm{SCI}}, \mathbf{Y}_{\mathrm{SCO}}, \mathbf{Y}_{\mathrm{SCR}}$, and $\mathbf{Y}_{\mathrm{SCT}}$ such that

$$
\begin{gathered}
\mathbf{I}_{I}=\mathbf{Y}_{\mathrm{SCI}} \mathbf{V}_{I}+\mathbf{Y}_{\mathrm{SCR}} \mathbf{V}_{O}, \\
\mathbf{I}_{O}=\mathbf{Y}_{\mathrm{SCT}} \mathbf{V}_{I}+\mathbf{Y}_{\mathrm{SCO}} \mathbf{V}_{O},
\end{gathered}
$$

where $\mathbf{I}_{I}$ is used to denote the column vector of the input currents, $\mathbf{I}_{O}$ is used to denote the column vector of the output currents, $\mathbf{V}_{I}$ is used to denote the column vector of the input voltages, and $\mathbf{V}_{O}$ is used to denote the column vector of the output voltages. We can also write

$$
\left(\begin{array}{c}
\mathbf{I}_{I} \\
\mathbf{I}_{O}
\end{array}\right)=\mathbf{Y}\left(\begin{array}{c}
\mathbf{V}_{I} \\
\mathbf{V}_{O}
\end{array}\right),
$$

where

$$
\mathbf{Y}=\left(\begin{array}{ll}
\mathbf{Y}_{\mathrm{SCI}} & \mathbf{Y}_{\mathrm{SCR}} \\
\mathbf{Y}_{\mathrm{SCT}} & \mathbf{Y}_{\mathrm{SCO}}
\end{array}\right)
$$

is the admittance matrix defined and used in Section 2. If the output ports of the MIPMOP device are connected to an $m$-port load presenting the impedance matrix $\mathbf{Z}_{L}$, the input ports of the MIPMOP device present an admittance matrix which may referred to as the loaded input admittance matrix and is given by

$$
\begin{aligned}
\mathbf{Y}_{\mathrm{LI}} & =\mathbf{Y}_{\mathrm{SCI}}-\mathbf{Y}_{\mathrm{SCR}}\left(\mathbf{1}_{m}+\mathbf{Z}_{L} \mathbf{Y}_{\mathrm{SCO}}\right)^{-1} \mathbf{Z}_{L} \mathbf{Y}_{\mathrm{SCT}} \\
& =\mathbf{Y}_{\mathrm{SCI}}-\mathbf{Y}_{\mathrm{SCR}} \mathbf{Z}_{L}\left(\mathbf{1}_{m}+\mathbf{Y}_{\mathrm{SCO}} \mathbf{Z}_{L}\right)^{-1} \mathbf{Y}_{\mathrm{SCT}}
\end{aligned}
$$

If the input ports of the MIPMOP device are connected to an $n$-port source presenting the impedance matrix $\mathbf{Z}_{S}$, the output ports of the MIPMOP device present an admittance matrix which may be referred to as the loaded output admittance matrix and is given by

$$
\begin{aligned}
\mathbf{Y}_{\mathrm{LO}} & =\mathbf{Y}_{\mathrm{SCO}}-\mathbf{Y}_{\mathrm{SCT}}\left(\mathbf{1}_{n}+\mathbf{Z}_{\mathrm{S}} \mathbf{Y}_{\mathrm{SCI}}\right)^{-1} \mathbf{Z}_{\mathrm{S}} \mathbf{Y}_{\mathrm{SCR}} \\
& =\mathbf{Y}_{\mathrm{SCO}}-\mathbf{Y}_{\mathrm{SCT}} \mathbf{Z}_{S}\left(\mathbf{1}_{n}+\mathbf{Y}_{\mathrm{SCI}} \mathbf{Z}_{S}\right)^{-1} \mathbf{Y}_{\mathrm{SCR}}
\end{aligned}
$$

The real power $P$ received by the linear MIPMOP device is given by

$$
P=\frac{1}{2}\left(\begin{array}{c}
\mathbf{V}_{I} \\
\mathbf{V}_{O}
\end{array}\right)^{*}\left(\mathbf{Y}+\mathbf{Y}^{*}\right)\left(\begin{array}{c}
\mathbf{V}_{I} \\
\mathbf{V}_{O}
\end{array}\right) .
$$

Thus, the MIPMOP device is lossless if and only if

$$
\begin{aligned}
\mathbf{Y}_{\mathrm{SCI}} & =-\mathbf{Y}_{\mathrm{SCI}}^{*}, \\
\mathbf{Y}_{\mathrm{SCO}} & =-\mathbf{Y}_{\mathrm{SCO}}^{*}, \\
\mathbf{Y}_{\mathrm{SCT}} & =-\mathbf{Y}_{\mathrm{SCR}}^{*} .
\end{aligned}
$$

If we assume that the linear MIPMOP device provides hermitian matching at the input ports, by (A.4) we get

$$
\mathbf{Z}_{S}^{*-1}=\mathbf{Y}_{\mathrm{SCI}}-\mathbf{Y}_{\mathrm{SCR}}\left(\mathbf{1}_{m}+\mathbf{Z}_{L} \mathbf{Y}_{\mathrm{SCO}}\right)^{-1} \mathbf{Z}_{L} \mathbf{Y}_{\mathrm{SCT}} .
$$

If we assume that $\mathbf{Z}_{S}{ }^{*-1}-\mathbf{Y}_{\mathrm{SCI}}$ is invertible, we get

$$
\mathbf{Z}_{L}^{-1}=-\mathbf{Y}_{\mathrm{SCO}}-\mathbf{Y}_{\mathrm{SCT}}\left(\mathbf{Z}_{S}^{*-1}-\mathbf{Y}_{\mathrm{SCI}}\right)^{-1} \mathbf{Y}_{\mathrm{SCR}}
$$

If we now assume that the MIPMOP device is lossless, by (A.7) we get

$$
\mathbf{Z}_{L}^{*-1}=\mathbf{Y}_{\mathrm{SCO}}-\mathbf{Y}_{\mathrm{SCT}}\left(\mathbf{1}_{n}+\mathbf{Z}_{S} \mathbf{Y}_{\mathrm{SCI}}\right)^{-1} \mathbf{Z}_{S} \mathbf{Y}_{\mathrm{SCR}}
$$

If we compare (A.5) and (A.10), we find the following theorem on simultaneous hermitian matching at the input ports and at the output ports (bilateral hermitian matching) of a lossless device: if $\mathbf{Z}_{S}^{*-1}-\mathbf{Y}_{\mathrm{SCI}}$ is invertible, hermitian matching at the input ports entails hermitian matching at the output ports. By symmetry, if $\mathbf{Z}_{S}^{*-1}-\mathbf{Y}_{\mathrm{SCO}}$ is invertible, hermitian matching at the output ports entails hermitian matching at the input ports.

We note that this theorem does not use reciprocity.

\section{References}

[1] H. Rothe and W. Dahlke, "Theory of noisy fourpoles," Proceedings of the IRE, vol. 44, no. 6, 1956.

[2] K. Hartmann, "Noise characterization of linear circuits," IEEE Transactions on Circuits and Systems, vol. 23, no. 10, pp. 581590, 1976. 
[3] R. Vaughan and N. Scott, "Closely spaced terminated monopoles for vehicular diversity antennas," in Proceedings of the Antennas and Propagation Society International Symposium, vol. 2, pp. 1093-1096, July 1992.

[4] J. W. Wallace, M. A. Jensen, A. L. Swindlehurst, and B. D. Jeffs, "Experimental characterization of the MIMO wireless channel: Data acquisition and analysis," IEEE Transactions on Wireless Communications, vol. 2, no. 2, pp. 335-343, 2003.

[5] J. P. Weem and Z. Popović, "A method for determining noise coupling in a phased array antenna," in Proceedings of the International Microwave Symposium Digest IEEE-MTT-S, pp. 271-274, May 2001.

[6] C. Craeye, B. Parvais, and X. Dardenne, "MoM simulation of signal-to-noise patterns in infinite and finite receiving antenna arrays," IEEE Transactions on Antennas and Propagation, vol. 52, no. 12, pp. 3245-3256, 2004.

[7] K. F. Warnick and B. D. Jeffs, "Efficiencies and system temperature for a beamforming array," IEEE Antennas and Wireless Propagation Letters, vol. 7, Article ID 2001752, pp. 565-568, 2008.

[8] M. V. Ivashina, R. Maaskant, and B. Woestenburg, "Equivalent system representation to model the beam sensitivity of receiving antenna arrays," IEEE Antennas and Wireless Propagation Letters, vol. 7, Article ID 2006917, pp. 733-737, 2008.

[9] K. F. Warnick, M. V. Ivashina, R. Maaskant, and B. Woestenburg, "Unified definitions of efficiencies and system noise temperature for receiving antenna arrays," IEEE Transactions on Antennas and Propagation, vol. 58, no. 6, Article ID 5439763, pp. 2121-2125, 2010.

[10] R. A. Speciale, "Advanced design of phased-array beam-forming networks," IEEE Antennas and Propagation Magazine, vol. 38, no. 4, pp. 22-34, 1996.

[11] S. Stein, "On cross coupling in multiple-beam antennas," IEEE Transactions on Antennas and Propagation, vol. 10, no. 5, pp. 548-557, 1962.

[12] C. A. Desoer, "The maximum power transfer theorem for $n$ ports," IEEE Transactions on Circuit Theory, vol. 20, no. 3, pp. 328-330, 1973.

[13] J. W. Wallace and M. A. Jensen, "Termination-dependent diversity performance of coupled antennas: network theory analysis," IEEE Transactions on Antennas and Propagation, vol. 52, no. 1, pp. 98-105, 2004.

[14] J. W. Wallace and M. A. Jensen, "Mutual coupling in MIMO wireless systems: a rigorous network theory analysis," IEEE Transactions on Wireless Communications, vol. 3, no. 4, pp. 1317-1325, 2004.

[15] M. T. Ivrlač and J. A. Nossek, "Toward a circuit theory of communication," IEEE Transactions on Circuits and Systems I, vol. 57, no. 7, Article ID 5446312, pp. 1663-1683, 2010.

[16] M. L. Morris and M. A. Jensen, "Network model for MIMO systems with coupled antennas and noisy amplifiers," IEEE Transactions on Antennas and Propagation, vol. 53, no. 1, pp. 545-552, 2005.

[17] J. Weber, C. Volmer, K. Blau, R. Stephan, and M. A. Hein, "Miniaturisation of antenna arrays for mobile communications," in Proceedings of the 35th European Microwave Conference, pp. 1173-1176, Paris, France, October 2005.

[18] J. Weber, C. Volmer, K. Blau, R. Stephan, and M. A. Hein, "Miniaturized antenna arrays using decoupling networks with realistic elements," IEEE Transactions on Microwave Theory and Techniques, vol. 54, no. 6, pp. 2733-2740, 2006.
[19] F. Broydé and E. Clavelier, "Taking advantage of mutual coupling in radio communication systems using a multiport antenna array," IEEE Antennas and Propagation Magazine, vol. 49, no. 4, pp. 208-220, 2007.

[20] U.S. patent no. 7,983,645, "Method and device for radio reception using a plurarity of antennas," Priority: French patent application 06/06502 of 18, 2006.

[21] H. A. Haus and R. B. Adler, Circuit Theory of Linear Noisy Networks, John Wiley \& Sons, New York, NY, USA, 1959.

[22] J. Randa, "Noise characterization of multiport amplifiers," IEEE Transactions on Microwave Theory and Techniques, vol. 49, no. 10, pp. 1757-1763, 2001.

[23] F. Broydé and E. Clavelier, "Signal and noise analysis of an MIMO-SSFA," IEEE Transactions on Circuits and Systems II, vol. 56, no. 6, pp. 439-443, 2009.

[24] H. Hillbrand and P. H. Russer, "An efficient method for computer aided noise analysis of linear amplifier networks," IEEE Transactions on Circuits and Systems, vol. 23, no. 4, pp. 235-238, 1976.

[25] L. Moura, P. P. Monteiro, and I. Darwazeh, "Generalized noise analysis technique for four-port linear networks," IEEE Transactions on Circuits and Systems I, vol. 52, no. 3, pp. 631640, 2005.

[26] R. A. Horn and C. R. Johnson, Matrix Analysis, Cambridge University Press, Cambridge, UK, 1985.

[27] U.S. patent no. 7,642,849, "Multiple-input and multipleoutput amplifier," Priority: French patent application 06/00388 of 17, 2006.

[28] U.S. patent no. 7,940,119, "Multiple-input and multipleoutput amplifier using mutual induction in the feedback network," Priority: French patent application 06/05633 of 23, 2006.

[29] F. Broydé and E. Clavelier, "Multiple-input-port and multipleoutput-port amplifier for wireless receivers," in Proceedings of the SAME Forum, Antipolis, France, October 2007.

[30] J. Engberg, "Simulteneous input power match and noise optimization using feedback," in Proceedings of the 4th European Microwave Conference, pp. 385-389, Montreux, Switzerland, September 1974.

[31] R. E. Lehmann and D. D. Heston, "X-band monolithic series feedback LNA," IEEE Transactions on Microwave Theory and Techniques, vol. 33, no. 12, pp. 1560-1566, 1985.

[32] K.-J. Sun, Z.-M. Tsai, K.-Y. Lin, and H. Wang, "A noise optimization formulation for CMOS low-noise amplifiers with on-chip low-Q inductors," IEEE Transactions on Microwave Theory and Techniques, vol. 54, no. 4, pp. 1554-1559, 2006.

[33] L. Belostotski and J. W. Haslett, "Noise figure optimization of inductively degenerated CMOS LNAs with integrated gate inductors," IEEE Transactions on Circuits and Systems I, vol. 53, no. 7, pp. 1409-1422, 2006. 

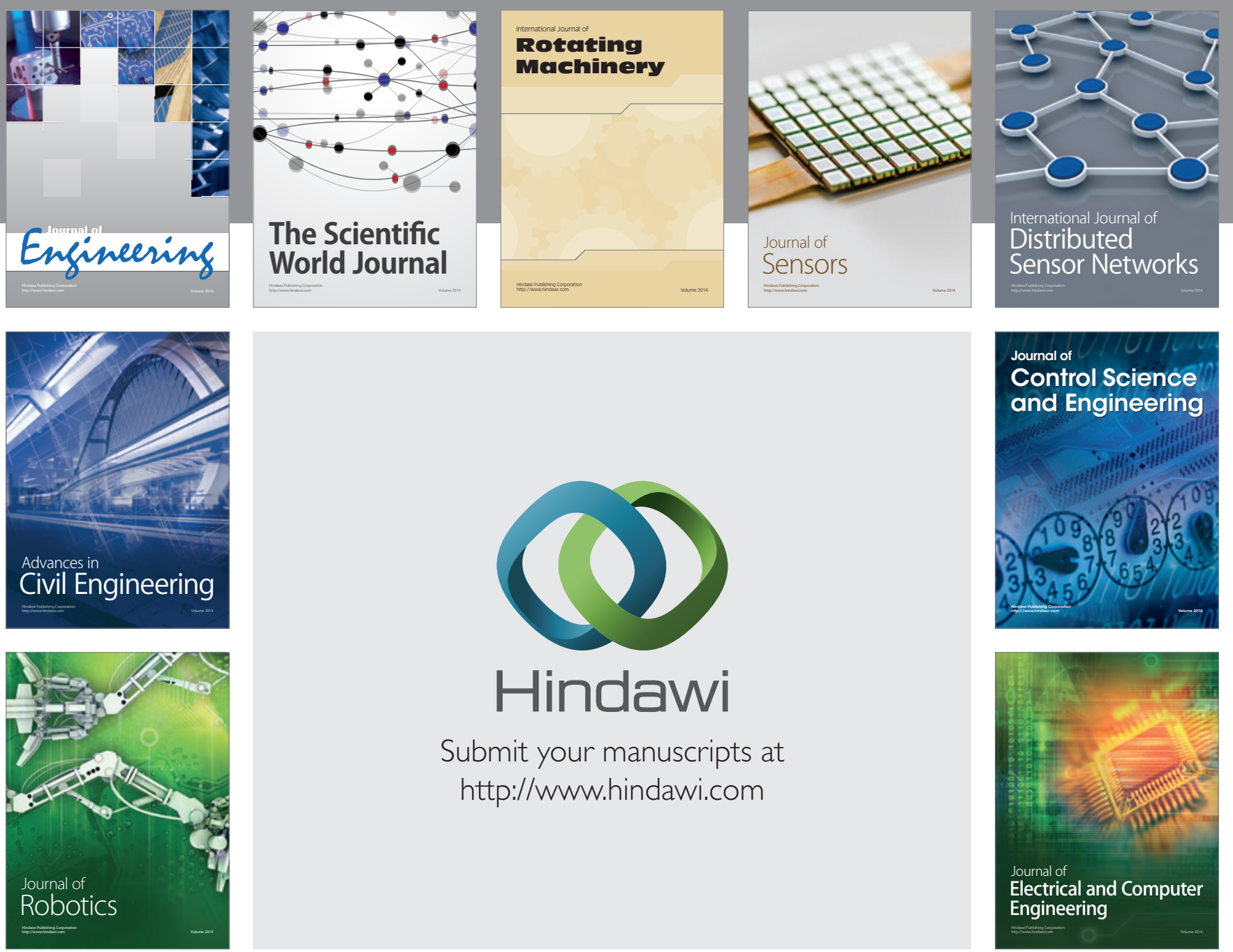

Submit your manuscripts at

http://www.hindawi.com
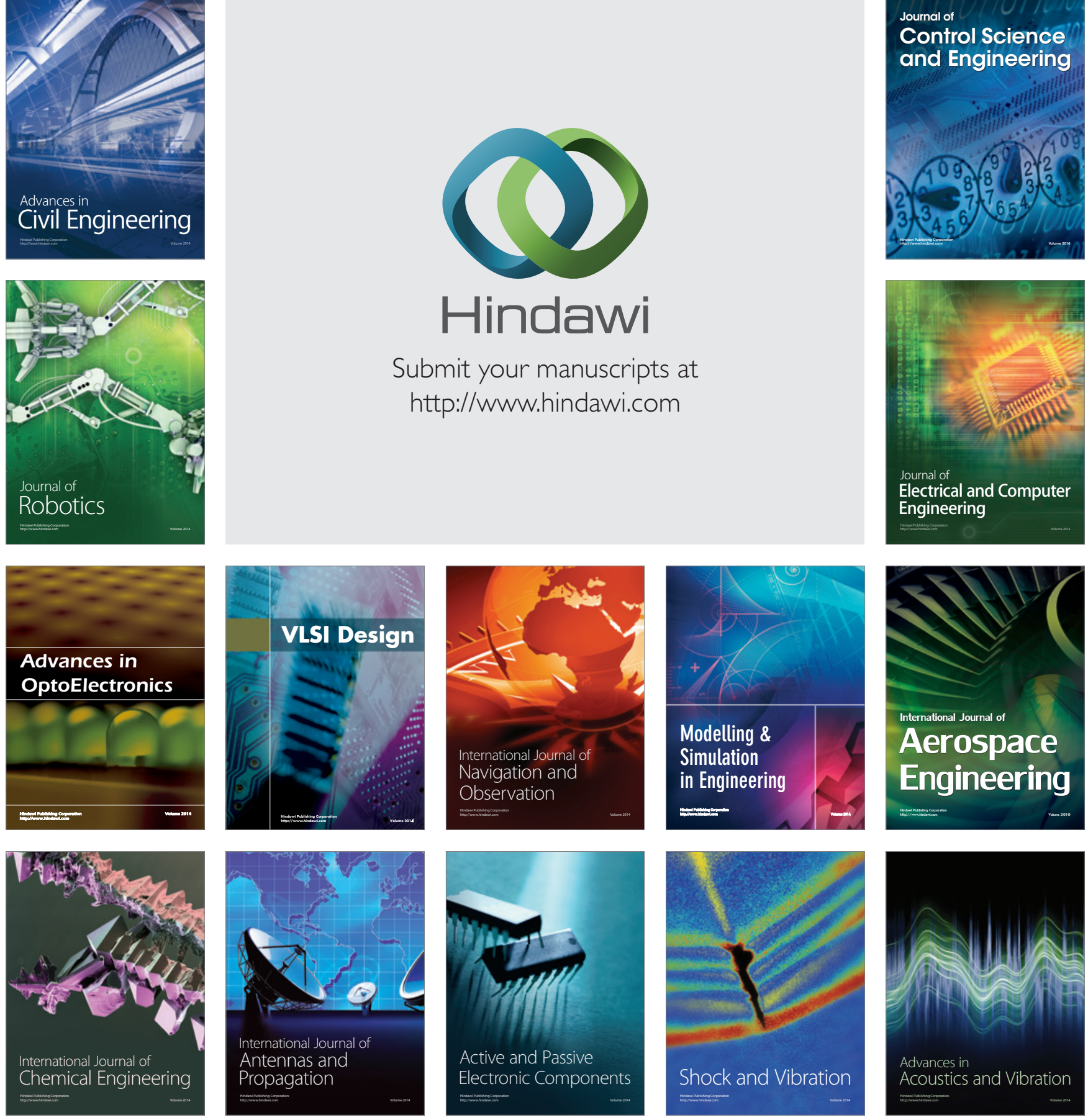